\title{
Recognition of Physical Activity between Physical Therapy and Non-Physical Therapy Students: Cross-Sectional Survey
}

\author{
Heun-Jae Ryu', Jung-Won Kwon², Young-Min Lee' \\ 'Department of Public Health Sciences, Graduate School, Dankook University, Cheonan, Republic of Korea; ${ }^{2}$ Department of Physical Therapy, College \\ of Health and Welfare Sciences, Dankook University, Cheonan, Republic of Korea
}

Purpose: This study was to the investigate recognition of physical activity between physical therapy students (PTS) and non-physical therapy students (NPTS) by measuring the level of physical activity using International Physical Activity Questionnaires (IPAQ).

Methods: A cross-sectional survey was completed by 191 university students. The IPAO with an additional question (Is physical activity necessary for your future job?) was used to evaluate the recognition and the amount of physical activity. The collected data were calculated as MET-minutes scores and were classified as walking, moderate, and vigorous level of physical activity. The students were analyzed by dividing them into those who had a part-time employment (16 PTS and 12 NPTS) and those who did not have a part-time employment (80 PTS and 83 NPTS).

Results: In students with a part-time employment, no significances were observed between the PTS and NPTS, in terms of MET, frequency and time of physical activity, and sitting time ( $p>0.05)$. In students without a part-time employment, the NPTS was significantly higher than the PTS for the MET and frequency of physical activity in a vigorous level $(p<0.05)$, and there were no significant differences in other levels of physical activity ( $p>0.05)$. In the additional question, the PTS showed a slightly higher than the NPTS $(p<0.05)$.

Conclusion: The physical therapy students did not remarkable barrier to recognition of physical activity, but there was a difference in their recognition of the vigorous level of physical activity. Therefore, the understanding of physical activity for PTS would play an important role in the recognition of how physical activity can be promoted.

Keywords: Physical activity, IPAQ, Cross-sectional survey, Physical therapy students

\section{INTRODUCTION}

Physical activity is defined as bodily movements that increase energy expenditure by contracting skeletal muscles. ${ }^{1}$ This is one of the critical energy consumption factors for maintaining energy balance. According to the World Health Organization (WHO), a lack of physical activity is the $4^{\text {th }}$ highest risk factor for mortality worldwide. ${ }^{2}$ Increased physical activity is a major factor in preventing adult diseases. ${ }^{3}$ Any physical activity in leisure time rather than a sedentary lifestyle provides significant health benefits. ${ }^{4,5}$

Recently, there has been a lack of exercise because of sedentary lifestyles. In a domestic statistical survey between 2014 and 2019, the physical activity decreased by $10.7 \%$, and the sedentary time increased by 0.8 hours. ${ }^{6,7}$ In addition, the Ministry of Health and Welfare of Korea reported that the proportion of the increase in the total, male and female adults with $\mathrm{BMI}=$
$25 \mathrm{~kg} / \mathrm{m}^{2}$ increased by $3.7 \%, 5 \%$, and $2.2 \%$, respectively, over the past four years, ${ }^{6}$ showing an increasing trend of metabolic syndrome, which accelerates geriatric diseases and physical deterioration. ${ }^{1}$

College students are in early adulthood, which is the healthiest period in their life cycle according to the measured mortality and morbidity, ${ }^{8}$ a critical period for establishing healthy lifestyles to prevent adult diseases and promote health in middle age. ${ }^{9}$ In addition, the possibility of lifestyle modification is high because the lifestyle in college students has not been established with respect to the health promotion habits compared to adults. ${ }^{10}$ An early-established healthy lifestyle in adulthood is continued until middle age, which is effective in preventing cardiovascular diseases and other adult diseases. ${ }^{10}$ On the other hand, college students have low physical activity and health because of their irregular lifestyles. ${ }^{11}$

Physical therapy is concerned with developing and maintaining move-
Received Nov 16, 2021 Revised Dec 20, 2021

Accepted Dec 21, 2021

Corresponding author Young-Min Lee

E-mail rickryu2321@gmail.com
Copylight ( 2021 The Korean Society of Physical Therapy

This is an Open Access article distribute under the terms of the Creative Commons Attribution Non-commercial License (https:// creativecommons.org/license/by-nc/4.o.) which permits unrestricted non-commercial use, distribution, and reproduction in any medium, provided the original work is properly cited. 
ment and functional abilities as much as possible throughout life and identifies and maximizes the movement possibilities within the areas of promotion, prevention, treatment, and rehabilitation. This includes both the process of evaluating the movement potential and establishing the treatment goals using the therapist's specialized knowledge and skills. ${ }^{12}$ Physical therapists have the knowledge and capabilities to inspire physical activity. They are positioned as a job group that can inspire patients and the public to undergo physical activity through clinical counseling and treatment. ${ }^{13}$ Physical therapy students learn the competency, recognition, and knowledge of physical activity related to health management compared to students of other majors. In addition, according to recognition of physical activity, physical therapy students are believed to have adequate knowledge and skills to undertake a role in physical activity promotion and their practice setting is deemed a feasible avenue for promoting an active lifestyle. Therefore, physical therapy students are exposed to an environment where they can be interested in performing physical activities and can have a high level of recognition of physical activity compared to other majors.

The International Physical Activity Questionnaires (IPAQ) measure physical activity in various situations, languages, and cultures. The questionnaire asks about the level of physical activity over the past week and has high reliability and validity. ${ }^{14}$ On the other hand, few studies used IPAQ to examine the level of physical activity recognition compared to physical therapy students and other students. Therefore, this study examined the recognition of physical activity between physical therapy and non-physical therapy students by measuring the level of physical activity using IPAQ.

\section{METHODS}

\section{Subjects}

Two hundred university students from D University located in Cheonan participated in this study, and 9 were dropped out. One hundred ninetyone students were divided into a physical therapy student group (PTS, $n=96$ ) and a non-physical therapy student group (NPTS, n=95). All subjects were given an overview of the study purpose and the procedures involved, and they provided written consent to participate. Ethical approval was obtained from the local university research ethics committee.

\section{Measurement}

\section{1) Korean version of $I P A Q$}

The IPAQ assesses all physical activities performed in a comprehensive range and evaluates the number of days of walking, moderate-intensity, and vigorous-intensity physical activity over the past week. Information on frequency (measured in days per week) and duration (time per day) are collected. ${ }^{15}$ The items were structured to provide separate scores on walking, moderate-intensity, and vigorous-intensity activity, as well as a combined total score to describe the overall level of activity. The total score was calculated as the sum of the duration (minutes) and frequency (days) of walking, moderate-intensity, and vigorous-intensity activities. Another measure of activity was calculated by weighting each activity type according to its energy requirements, as defined in METs (MET is a multiple of the resting metabolic rate), yielding a score in MET-minutes. The MET value is based on the MET score for each activity type reported in the IPAQ reliability study conducted in 2000-2001 and previous studies. The average MET score was obtained. ${ }^{16}$ The IPAQ scoring method is presented through guidelines divided into three categories.

\section{a. Inactive (Category 1 )}

This is the lowest level of physical activity. Individuals who do not meet the criteria for Categories 2 or 3 are considered 'insufficiently active'.

\section{b. Minimally Active (Category 2)}

The minimum pattern of activity to be classified as "sufficiently active" is any one of the following three criteria.

(1) Three or more days of vigorous activity of at least 20 minutes per day, or

(2) Five or more days of moderate-intensity activity or walking of at least 30 minutes per day, or

(3) Five or more days of any combination of walking, moderate-intensity or vigorous-intensity activities achieving a minimum of at least $600 \mathrm{MET}-\mathrm{min} /$ week.

c. Health-enhancing physical activity (HEPA) Active (Category 3)

The two criteria for classification as "HEPA active" are

(1) Vigorous-intensity activity on at least three days, achieving a minimum of at least 1,500 MET-minutes/week, or

(2) Seven or more days of any combination of walking, moderate-intensity, or vigorous-intensity activities, achieving a minimum of at least 3,000 MET-minutes/week. 


\section{2) Metabolic Equivalent Task (MET)}

The MET score (metabolic equivalent of task score) was used as one of the methods to measure the intensity of physical activity when measuring the level of physical activity based on the experience in health and health. ${ }^{17}$ Using the MET score, the physical activity could be classified as dynamic or static activity based on the energy metabolism. When sitting, the MET score is 1; 1 MET means an oxygen consumption of $2.5 \mathrm{~mL} / \mathrm{O}^{2} / \mathrm{kg}^{\star} \mathrm{min}$ per minute. Based on the MET score in a previous study, ${ }^{18}$ physical activity was divided into two major parts, dynamic and static, and subdivided into four subunits. A MET score of six or more means vigorous activity, and a MET score of three or more and less than six indicates moderate activity. These two activities consume a large amount of oxygen and correspond to dynamic activities. The remaining static activities with a score of less than three are sedentary activities. Activities other than sedentary activities with a score of less than three are classified as light activities. ${ }^{19}$

\section{3) MET calculation}

The data collected in this study were calculated as the MET-minutes scores based on the conversion method to IPAQ scores. It is a value obtained by multiplying the reference MET value for each physical activity by the number of days of the corresponding activity in minutes and the corresponding activity time per day in minutes. The points to consider in scoring are as follows. All walking, moderate and vigorous physical activity must not exceed 16 hours. If walking, moderate, or vigorous physical activity is recorded as more than 240 minutes, it is calculated by adjusting it to 240. In addition, physical activity for less than 10 minutes is considered not performed. The method for calculating a MET unit for how many minutes each corresponding physical activity was performed in a week is as follows: ${ }^{15}$

(1) Walking activity MET-min/week $=3.3 \times$ min of activity/day $\times$ days perweek;

(2) Moderate activity MET-min/week $=4.0 \times$ min of activity/day $\times$ days perweek;

(3) Vigorous activity MET-min/week $=8.0 \times$ min of activity $/$ day $\times$ days per week;

(4) Total amount of physical activity= walking activity MET-min/ week + moderate activity MET-min/week + vigorous activity MET$\mathrm{min} /$ week.

\section{4) Additional questionnaires}

The question "Is physical activity (exercise) necessary for your future job?" was added to investigate the recognition of the necessity of physical activity, and the reason why physical activity is necessary was conducted in a multiple-choice format except for the subjective format of the 5th line. In addition, subjects were asked to write subjectively about why physical activity was unnecessary.

\section{Experimental procedure}

The amount of physical activity was measured using the IPAQ for 191 university students. The recommended amount of vigorous, moderate, and light activity of dynamic activity was presented through a questionnaire. This study investigated how many days each activity was performed in a week. For example, structured questions were used: 'In the past seven days, on how many days did you engage in vigorous physical activity for more than 10 minutes? (an activity that involves strenuous physical exertion or that makes breathing much more difficult than usual. For example, moving heavy luggage, basketball, soccer, hiking, biking, running, and longdistance bike riding); 'How many minutes on one of those days do you usually spend doing vigorous physical activity?; "Is physical activity (exercise) necessary for your future job?". Based on the subjective judgment of the respondent, the time spent in the activity was recorded in a short-answer format.

The collected data were calculated as the MET-minutes scores and converted to IPAQ scores based on the conversion method. The students were analyzed by dividing them into those who had a part-time employment (16 physical therapy and 12 non-physical therapy students) and those who did not (80 physical therapy and 83 non-physical therapy students). Each group was divided into criteria corresponding to the three physical activity categories from IPAQ. In addition, this study compared the total amount of physical activity, including the MET of each activity, times per day of each activity, and the number of days of vigorous, moderate, and walking activity between physical therapy and non-physical therapy students.

\section{Data analysis}

The data collected in this study were encoded and analyzed using the Windows SPSS version 25.0 (IBM Co., USA) program. Descriptive statistics were performed on the general characteristics of the study subjects, such as age, gender, and major. A Kolmogorov-Smirnov test was used for the normality test among the outcome measures. The gender ratio by major, the number of students (with or without a part-time employment), the 
three categories ratio of physical activity from IPAQ, and the answer to the question of 'Is physical activity exercise necessary for the future job?' were analyzed using Chi-square tests. A Mann-Whitney test was performed to compare the differences between the physical therapy and non-physical therapy students in terms of time per day, day per week, and MET of each category. A statistical significance level was set to $\mathrm{p}<0.05$.

\section{RESULTS}

\section{General characteristics}

Table 1 lists the demographic data for the two groups. The overall number of students between majors was 191: 96 for the PTS and 95 for the NPTS.
The age, gender distribution, and IPAQ category were similar in the two groups ( $p>0.05$ ). On the other hand, there was a significant difference in an additional questionnaire $(\mathrm{p}<0.05)$.

\section{IPAQ outcomes in students with a part-time employment}

Table 2 lists the calculated MET parameters in each physical activity level. The MET, frequency and time of physical activity, and sitting time were similar in the two groups $(\mathrm{p}>0.05)$.

\section{IPAQ outcomes in students without a part-time employment}

Table 3 lists the calculated MET parameters in each physical activity level. In the Vigorous level, the MET and frequency of physical activity was sig-

Table 1. General characteristics

\begin{tabular}{|c|c|c|c|c|c|}
\hline Variable & Part-time Employment & Category & PTS $(N=96)$ & NPTS $(N=95)$ & $p$ \\
\hline \multirow[t]{2}{*}{ Age (yr) } & Yes & & $22.13 \pm 1.50$ & $22.25 \pm 1.14$ & 0.755 \\
\hline & No & & $22.40 \pm 1.73$ & $22.48 \pm 1.80$ & 0.946 \\
\hline \multirow[t]{4}{*}{ Gender (n) } & Yes & Male & 4 & 3 & 1.000 \\
\hline & & Female & 12 & 9 & \\
\hline & No & Male & 28 & 40 & 0.088 \\
\hline & & Female & 52 & 43 & \\
\hline \multirow[t]{6}{*}{ IPAQ category (\%) } & Yes & Inactive & 5.23 & 4.19 & 0.155 \\
\hline & & Minimally Active & 3.14 & 1.05 & \\
\hline & & HEPA Active & 0 & 1.05 & \\
\hline & No & Inactive & 21.98 & 18.84 & 0.186 \\
\hline & & Minimally Active & 13.61 & 13.08 & \\
\hline & & HEPA Active & 6.28 & 11.51 & \\
\hline \multirow[t]{2}{*}{ Additional questionnaire (n) } & Yes & & 95 & 87 & $0.016^{*}$ \\
\hline & No & & 1 & 8 & \\
\hline
\end{tabular}

Mean \pm standard deviation.

PTS: Physical therapy students' group, NPTS: Non-physical therapy students' group, IPAQ: International physical activity questionnaires.

${ }^{*} \mathrm{p}<0.05$.

Table 2. Comparison of physical activity in students with a part-time employment between PTS and NPTS

\begin{tabular}{|c|c|c|c|c|}
\hline & Levels & $\operatorname{PTS}(n=16)$ & NPTS $(n=12)$ & $p$ \\
\hline \multirow[t]{4}{*}{ MET (MET-min/week) } & Walking & $582.65 \pm 382.89$ & $1,189.93 \pm 2,606.75$ & 0.209 \\
\hline & Moderate & $156.25 \pm 358.68$ & $266.67 \pm 577.22$ & 0.979 \\
\hline & Vigorous & $635.00 \pm 1,311.76$ & $3,140.00 \pm 7,131.24$ & 0.713 \\
\hline & Total & $1,373.91 \pm 1,862.95$ & $4,596.59 \pm 8,990.95$ & 0.444 \\
\hline \multirow[t]{3}{*}{ Frequency (days per week) } & Walking & $4.19 \pm 2.20$ & $4.42 \pm 2.75$ & 0.654 \\
\hline & Moderate & $0.88 \pm 1.20$ & $0.92 \pm 1.56$ & 0.731 \\
\hline & Vigorous & $1.25 \pm 1.13$ & $1.83 \pm 2.66$ & 0.804 \\
\hline \multirow[t]{3}{*}{ Time (minutes per day) } & Walking & $48.44 \pm 53.91$ & $45.92 \pm 69.81$ & 0.203 \\
\hline & Moderate & $16.56 \pm 30.26$ & $20.83 \pm 34.23$ & 0.937 \\
\hline & Vigorous & $32.19 \pm 59.78$ & $57.08 \pm 99.51$ & 0.556 \\
\hline Sitting time (minutes per day) & Total & $21.31 \pm 30.62$ & $25.25 \pm 35.25$ & 0.870 \\
\hline
\end{tabular}

Mean \pm standard deviation.

PTS: Physical therapy students' group, NPTS: Non-physical therapy students' group. 
Table 3. Comparison of physical activity in students without a part-time employment between PTS and NPTS

\begin{tabular}{llccc}
\hline & Levels & PTS $(n=80)$ & NPTS $(n=83)$ & $p$ \\
\hline MET (MET-min/week) & Walking & $846.66 \pm 1431.11$ & $749.26 \pm 1001.51$ & 0.910 \\
& Moderate & $340.75 \pm 492.81$ & $472.82 \pm 687.79$ & 0.105 \\
& Vigorous & $593.00 \pm 1,018.21$ & $1,133.69 \pm 1,631.52$ & $0.017^{\star}$ \\
Frequency (days per week) & Total & $1,780.41 \pm 1,955.29$ & $2,355.77 \pm 2,577.34$ & 0.146 \\
& Walking & $4.58 \pm 2.07$ & $4.64 \pm 2.11$ & 0.780 \\
& Moderate & $1.88 \pm 1.86$ & $2.37 \pm 2.09$ & 0.129 \\
Time (minutes per day) & Vigorous & $1.43 \pm 1.91$ & $2.34 \pm 2.29$ & $0.009^{*}$ \\
& Walking & $45.44 \pm 44.42$ & $40.54 \pm 38.67$ & 0.456 \\
Sitting time (minutes per day) & Moderate & $27.15 \pm 33.88$ & $33.51 \pm 34.07$ & 0.132 \\
\hline
\end{tabular}

Mean \pm standard deviation

PTS: Physical therapy students' group, NPTS: Non-physical therapy students' group. ${ }^{*} \mathrm{p}<0.05$.

nificantly higher in the NPTS than the PTS ( $\mathrm{p}<0.05)$, but other levels were similar in the two groups $(\mathrm{p}>0.05)$. Furthermore, the physical activity and sitting times were similar in the two groups $(\mathrm{p}>0.05)$.

\section{Additional questionnaires for the necessity of physical activity}

In the PTS, out of 95 students who answered 'Yes', 79 students answered that self-management is necessary for work. Thirteen students answered that knowledge and experience gained through physical activity (exercise) are necessary for future jobs. Two students answered that physical activity is needed to increase the competitiveness over others (e.g., salary, promotion, and incentive), and one student answered that a healthy body shape is helpful for managing the health of others.

In the NPTS, out of 87 students who answered 'Yes', 75 students answered that self-management is necessary for work. Eight students answered that the knowledge and experience gained through physical activity (exercise) are necessary for future employment. Four students who answered 'To increase competitiveness over others (e.g., salary, promotion, and incentive)'.

As to the reason why physical activity is not required for future employment, one student in the PTS answered, As the concept of physical space becomes meaningless according to the era of Untact (a new word meaning non-face-to-face), the meaning of the physical activity will also decrease'. In the NPTS, four students answered, 'I am currently healthy and do not need to exercise', and two students answered, 'I do not need to perform physical activity because there are many jobs that use computers'. Two students answered, 'I think I will spend more time sitting'.

\section{DISCUSSION}

The IPAQ, which measures the level of physical activity over the past week, was used to assess the recognition of physical activity between physical therapy and non-physical therapy students. The results showed no significant differences in MET, frequency and time of physical activity, and the sitting time between PTS and NPTS students with part-time employment. A previous study examined the relationship between employment and physical activity. ${ }^{20}$ Employment is positively associated with the activity levels, and the job type is a strong predictor of the daily activity levels in both genders. Therefore, the lack of a difference in recognition of physical activity among students with part-time employment is not surprising.

On the other hand, in the students without part-time employment, the NPTS was significantly higher than the PTS for the MET and frequency at the vigorous level of physical activity. A survey of physiotherapists in Ireland reported that only $51 \%$ of participants could state the current physical activity guidelines accurately. ${ }^{21}$ In addition, previous research found that only $16 \%$ of physical therapists were aware of the physical activity guidelines, while $62 \%$ were inactive. ${ }^{22}$ The explanations included a lack of time, a lack of belief in the effectiveness of short-term interventions, a lack of perceived knowledge, and the patient's sense of unacceptability. ${ }^{22}$ This highlights some knowledge gaps on the physical activity guidelines for health among many physical therapy students that need to be addressed. No studies have been examined the knowledge of physical activity guidelines among Korean physical therapy students. Another factor is that physical therapy education emphasizes theory rather than practice on the job. ${ }^{23}$ This education is widely acknowledged as a significant barrier to 
healthcare professionals properly promoting it. ${ }^{24}$ Hence, physical therapy students are inactive are because of a large amount of learning and insufficient time. In vigorous physical activity and days per week, the PTS was $593.00 \pm 1,018 .{ }^{21}$ MET-min/week and $1.43 \pm 1.91$ days, which was significantly lower than the NPTS mean of 1,133.69 $\pm 1,631.52$ MET-min/week and $2.34 \pm 2.29$ days. It was found that there was a significant difference in the vigorous physical activity compared to the NPTS. A survey of university students in Korea reported that natural science students had the highest physical activity according to major and greater physical activity levels than humanities, social sciences, business, and medicine students. ${ }^{25}$ This result supports the findings that physiotherapy students have lower physical activity levels because the curriculum in physical therapy is similar to that in medicine. In addition, physical therapy students tend to avoid vigorous activity and high-intensity exercises that have been shown to overload and adversely affect the joints of the body. ${ }^{12,26}$ Therefore, they choose low- or moderate-intensity exercises instead of high-intensity exercises.

In response to the question "Is physical activity required in the future?", the PTS showed a slightly more favorable attitude than the NPTS. Physical therapists are involved in the primary prevention of non-communicable diseases and are also involved in risk reduction for these diseases. These professionals are also more likely than other health personnel to be approached about physical fitness, physical activity, and exercise. The present results showed that physical therapy students have good knowledge of physical activity promotion messages and believe promoting physical activity is feasible for them. Physical activity-related subjects comprise a substantial part of the physical therapy curriculum. Physical therapy students are expected to learn the benefits of physical activity by completing courses that include specific knowledge related to physical activity, such as exercise therapy, therapeutic interventions, and exercise prescription. ${ }^{23} \mathrm{~A}$ previous study reported that physical therapy students had higher satisfaction with the physical therapy profession and a positive job recognition of physical therapy. ${ }^{27}$ Therefore, most students believe that physiotherapists should be physically active as role models to their patients, indicating that they are aware of the need to remain healthy and 'practice what they preach'. This means that physical therapy students are fully aware of the need for physical activity in the future. In addition, most of the students, regardless of their major, answered that they needed self-management to work. The benefits of physical activity are well known and well publicized. Generally, participation in any type or amount of physical activity can provide important health benefits compared to a sedentary lifestyle. Physically active exercise reduces the all-cause mortality risk significantly com- pared to insufficient activity ${ }^{28}$ and has also been shown to improve mental wellbeing by establishing emotional stability. ${ }^{29}$ During the past few years, there has been growing interest in the necessity of physical activity by increasing information on health issues. These results are believed to result from many students feeling that behavioral changes for a healthy lifestyle are necessary to find employment in the future.

Overall, the physical therapy students in the present study recognized the need for physical activity, but there was a difference in their recognition of the vigorous levels of physical activity. Hence, it can be argued that a lack of knowledge and information on the intensity of exercise that could confer health benefits, rather than a lack of time, or lack of interest, could restrict them from vigorous physical activity. In addition, the physical activity guidelines are insufficiently taught in undergraduate physical therapy curricula, and there is a lack of knowledge among physical therapy students. Therefore, developing physical activity guidelines for physical therapy students would play an important role in recognizing how physical activity can be promoted. This study had several limitations. First, only one university participated, and the number of participants was small. Second, this study did not include a homogeneous assessment of the students with part-time employment due to lack of HEPA activity. Third, the student's academic environment was not considered. Therefore, further study is needed to compensate for these limitations.

\section{REFERENCES}

1. Manley AF. Physical activity and health: a report of the surgeon general. Atlanta, DIANE Publishing, 1996:7-20.

2. World Health Organization. Global recommendations on physical activity for health. 2010:10.

3. Brayne H, Carr H, Goosey D. Law for social workers. Oxford, Oxford University Press, 2015;21(25):279.

4. US Department of Health and Human Services. 2010 healthy people: understanding and Improving health. 2nd ed. Washington DC, U.S. Government Printing Office, 2000:26-27.

5. Durstine JL, Gordon B, Wang Z et al. Chronic disease and the link to physical activity. J Sport Health Sci. 2013;2(1):3-11.

6. Statistcs Korea. Trend of physical activity practice rate, 2008-2019 and trend of obesity management level, 2007-2019. [Internet]. South Korea: 2021 April 22 [cited 2021 Nov 1] Available from: http://kdca.go.kr/ board/board.es? $\mathrm{mid}=\mathrm{a} 20602010000 \& \mathrm{bid}=0034 \&$ act $=$ view $\&$ list_ no $=713106$.

7. Statistcs Korea. Adult obesity rate and physical activity rate 2014-2018. [Internet]. South Korea: 2020 Oct 25 [cited 2021 Nov 1] Available from: http://www.mohw.go.kr/react/al/sal0301vw.jsp?PAR_MENU_ID= 04\&MENU_ID=0403\&CONT_SEQ=360437.

8. Helmer SM., Krämer A, Mikolajczyk RT. Health-related locus of control 
and health behaviour among university students in North Rhine Westphalia, Germany. BMC Res Notes. 2012;5(1):1-8.

9. Kim YS, Yoon IS, Lee WJ et al. Anthropometric measurements of the upper and lower body balance and exercise habit among female college students in some parts of the Gyeongnam. J Life Sci. 2010;20(4):535-42.

10. Kim H. Comparison of health behavior, stress and stress coping type between undergraduate nursing students and female students in other majors. J East-West Nurs Res. 2015;21(1):28-35.

11. Kim JP, Moon HS. The relation between wellness and life satisfaction of students' participation in university physical education class. J Leis Recreat Stud. 2008;32(4):5-19.

12. Song MY, Choi MH, Kim KM et al. The consideration of definition of physical therapy in South Korea. J Korean Soc Phys Med. 2010;5(2):13341.

13. Stubbs T, Vita P, Van der Ploeg H et al. Physical activity promotion: Are GPs getting the message? Aust Fam. 2007;36(10):871-4.

14. Hallal PC, Victora C. Reliability and validity of the international physical activity questionnaire (IPAQ). Med Sci Sports Exerc. 2004;36(3):556.

15. IPAQ Research Committee. Guidelines for data processing and analysis of the International Physical Activity Questionnaire (IPAQ)-short and long forms. [Internet]. 2004 April [cited 2021 Nov 21]. Available from: http://www.physio-pedia.com/images/c/c7/Quidelines_for_interpreting_the_IPAQ.pdf

16. Yoo JS, Chin JH, Kim MJ et al. College students' dietary behavior, healthrelated lifestyles and nutrient intake status by physical activity levels using international physical activity questionnaire (IPAQ) in Incheon area. J Nutr Health. 2008;41(8):818-31.

17. Ainsworth BE, Haskell WL, Whitt MC et al. Compendium of physical activities: an update of activity codes and met intensities. Med Sci Sports Exerc. 2000;32(9; SUPP/1):S498-S504.

18. Ainsworth BE, Haskell WL, Leon AS et al. Compendium of physical activities: classification of energy costs of human physical activities. Med
Sci Sports Exerc. 1993;25(1):71-80.

19. Tudor Locke C, Johnson WD, Katzmarzyk PT. Frequently reported activities by intensity for us adults: the american time use survey. Am J Prev Med. 2010;39(4):e13-20.

20. Van Domelen DR, Koster A, Caserotti P et al. Employment and physical activity in the US. Am J Prev Med. 2011;41(2):136-45.

21. Barrett EM, Darker CD, Hussey J. Promotion of physical activity in primary care: knowledge and practice of general practitioners and physiotherapists. J Public Health. 2013;21(1):63-9.

22. Lowe A, Littlewood C, McLean S et al. Physiotherapy and physical activity: a cross-sectional survey exploring physical activity promotion, knowledge of physical activity guidelines and the physical activity habits of UK physiotherapists. BMJ Open Sport Exerc Med. 2017;3(1):e000290.

23. Goo BO, Park MC, Lee MH et al. The review on physical therapy curriculum in South Korea. J Korean Soc Phys Med. 2010;5(2):165-72.

24. Dunlop M, Murray AD. Major limitations in knowledge of physical activity guidelines among UK medical students revealed: implications for the undergraduate medical curriculum. Br J Sports Med. 2013;47(11): 718-20.

25. Yang JS, Ko WJ, Yoo SH. The effects of physical activity on wellness in university students. J Korean Soc Living Environ Sys. 2014;21(6):102332.

26. Slater TF. Free radical mechanisms in tissue injury. Cell Funct Dis. 1988: 209-18.

27. Lee KJ. A study on the job recognition and career preference of physical therapy major college students. J Korean Phys Ther Sci. 2018;25(3):3242.

28. Cha JT, Jee YS. The effects of regular exercise on cardiovascular function and depression in elderly. J Korean Phys Edu. 2004;43(5):331-40.

29. Balady GJ. Acsm's guidelines for exercise testing and prescription. Baltimore, Lippincott Williams \& Wilkins, 2000:9. 